\title{
Chondroprotektiva (Glukosamin, Chondroitin) und Fischöl (Omega-3-FS): Wirkevidenz bei Erkrankungen des Bewegungsapparates
}

Der vorliegende Report der Arthritis Research Campaign ist ein Leitfaden, der einen Überblick gibt zur aktuellen wissenschaftlichen Beweislage beim Einsatz komplementärmedizinischer Arzneimittel bei Erkrankungen des Bewegungsapparates wie zum Beispiel Rheumatoide Arthritis RA, Arthrose (Osteoarthritis OA) und Fibromyalgie. Der Report bestätigt eine klare Wirksamkeit von Fischöl bei RA. Bei OA zeigt sich eine vielversprechende Beweislage zur Wirksamkeit von Chondroitin und Glukosaminsulfat und eine nicht überzeugende Beweislage zur Wirksamkeit von Glukosaminhydrochlorid.

Die Arthritis Research Campaign (ARC) ist einer der grössten Medizin-Verbände Englands. Seine Hauptaufgabe besteht in der Erforschung der Ursachen von entzündlichen und degenerativen Gelenkerkrankungen sowie anderer Formen muskuloskelettaler Erkrankungen. Mitglieder des Verbandes sind die renommierten Universitäten von Aberdeen, Exeter, Leeds, Liverpool, Plymouth und Southampton.

Der von der $A R C$ verfasste und herausgegebene Report Complementary and alternative medicines for the treatment of rheumatoid arthritis, osteoarthritis and fibromyalgia ist ein evidenzbasierter Report zum Einsatz von vierzig komplementärmedizinischen Arzneimitteln bei Arthritis und Erkrankungen des Bewegungsapparates. Er basiert auf den Ergebnissen randomisierter kontrollierter Studien (RCTs), ausgewählt nach dem Jadad-Score 3. Berücksichtigt wurden Studien, die entweder Standardmedikationen untereinander oder mit Placebo vergleichen. Experten auf dem Gebiet der Datenanalyse, Rheumatologie, Komplementärmedizin und Ernährungswissenschaft bewerteten die eingesetzten Arzneimittel nach ihrer Wirksamkeit mit den Noten 1 bis 5 (1: Die vorliegenden Studienergebnisse lassen den Schluss zu, dass die Substanz nicht wirksam ist; 5: Es liegen schlüssige Beweise für die Wirksamkeit vor). Die Wirksamkeit wird bewertet anhand von Schmerzlinderung, Verbesserung von Bewegungsfähigkeit/Mobilität und Steigerung des allgemeinen Wohlbefindens. Der Report ordnet Medikationen mit Hilfe eines Ampelsystems auch nach ihrer Sicherheit ein.

\section{Fischöl bei RA (Wirk-Score 5)}

Öle aus Fisch sind reich an essenziellen Omega-3-Fettsäuren, die ausgeprägte anti-inflammatorische Eigenschaften besitzen: Sie reduzieren signifikant die Freisetzung proinflammatorischer Mediatoren aus Leukozyten und sind beteiligt an der Synthese anti-inflammatorischer Substanzen.

Ein in jüngerer Zeit durchgeführter Review gibt einen Überblick über sämtliche Studienergebnisse aus insgesamt 17 RCTs, in denen die Rolle von Fischöl bei Patienten mit RA untersucht wurde. Die Dosis der in diesen Studien verwendeten Omega-3-Fettsäuren betrug zwischen 1,6 und 7,1 g/Tag mit einer mittleren Dosis von 3,5 g/Tag. Die Ergebnisse dieser Studien lassen den Schluss zu, dass FischölSupplemente generell sicher in der Anwendung sind und signifikant Gelenkschmerz lindern, die Dauer von Morgensteifigkeit reduzieren, Schmerz lindern und die Anzahl schmerzender, geschwollener Gelenke verringern können. Darüberhinaus vermag Fischöl die Anwendung von Schmerzmitteln bei Patienten mit RA signifikant zu verringern. Der Einsatz von Fischöl bei Patienten mit Osteoarthritis ist nicht ausreichend belegt.

Öle aus Fisch sind sicher und zeigen keine nennenswerten unerwünschten Wirkungen, wenn sie in therapeutischen Dosen eingenommen werden.

\section{Chondroitin bei OA (Wirk-Score 3)}

Chondroitin ist ein wesentlicher Bestandteil des Gelenkknorpels. Es verleiht dem Knorpel Elastizität, indem es hilft, Wasser zu speichern. Untersuchungen zeigen, dass Chondroitin die Aktivität von Substanzen, die Kollagen in den Gelenken zersetzen, reduzieren kann. Andere Studien deuten hin auf seine anti-inflammatorischen Eigenschaften sowie auf sein Potenzial, den Abbau von Gelenkknorpel zu verhindern und Reparaturmechanismen zu stimulieren.

Ein Review-Artikel aus jüngerer Zeit fasst die Ergebnisse aus 19 RCTs zusammen, bei denen die Wirksamkeit von Chondroitin an Patienten mit OA an Knie und Hüfte untersucht wurde. Die Anzahl der jeweiligen Studienteilnehmer bewegte sich zwischen 46 und 631 Patienten und die Studiendauer lag zwischen 13 und 132 Wochen. Sechzehn Studien verglichen den potentiellen Nutzen von Chondroitin mit dem eines Placebos und zwölf von diesen zeigten, dass Chondroitin bei der Schmerzlinderung dem Placebo signifikant überlegen war. Fünf Studien untersuchten die potentielle Wirkung von Chondroitin auf eine strukturelle Vergrösserung der Weite des Gelenkspalts. Die meisten dieser Studien kamen zu dem Schluss, dass Chondroitin hier eine geringe und nicht signifikante Wirkung im Vergleich zu

Wirk-Score zu den untersuchten Arzneimitteln

1 Es gibt insgesamt keinen Beweis, dass die Substanz wirkt; Beweise, dass sie nicht wirkt, sind stärker.

2 Es gibt nur einen geringen Beweis (meist eine Studie), dass die Substanz wirken könnte.

3 Es gibt vielsprechende Ergebnisse aus mehreren Studien, dass die Substanz wirkt.

4 Es besteht eine Konsistenz in den Ergebnissen aus mehr als einer Studie, die nahelegen, dass die Substanz wirksam ist.

5 Es gibt schlüssige Beweise aus mehreren Studien, dass die Substanz wirksam ist. 


\section{Glukosamin bei Gelenkbeschwerden}

Kontrollierte klinische Studien erlauben folgende Aussagen:

Glukosamin-Sulfat (1'500 mg/Tag):

- Vermindert Gelenkschmerzen bei Osteoarthritis.

- Vermindert Schmerzen bei Osteoarthritis der Kniegelenke.

- Schützt den Knorpel vor Schädigung/Verschleiss.

- Ist ein wichtiger Faktor zum Erhalt von gesunden Gelenken und gesundem Knorpel.

Glukosamin-Hydrochlorid (1'500-2'000 mg/Tag):

- Trägt bei zum Erhalt von gesunden Gelenken und Knorpel.

Health Canada Drug-Monograph Glucosamine, 19. Juni 2009

www.hc-sc.gc.ca/dhp-mps/prodnatur/applications/licen-prod/monograph/mono_glucosamine-eng.php

Placebo hat. Im Hinblick auf die Reduktion von Analgetika zeigten 12 (von 16) Studien eine signifikant bessere Wirksamkeit von Chondroitin im Vergleich zu Placebo.

Bei der überwiegenden Mehrheit der Studien aus diesem Review waren Anzahl und Schweregrad von unerwünschten Wirkungen bei Patienten unter Chondroitin geringer oder ähnlich wie bei Patienten unter Placebo.

\section{Glukosamin bei OA}

Glukosamin spielt eine wichtige Rolle bei der Synthese von Glykosaminoglykanen und Glykoproteinen, essenziellen Bausteinen vieler Gelenkstrukturen, einschliesslich Bänder, Sehnen, Knorpel und Synovialflüssigkeit. Tierversuche haben gezeigt, dass die Verabreichung von Glucosamin sowohl eine Degeneration des Gelenkknorpels verhindern als auch ihn wieder aufbauen kann.

Glukosamin ist eine der am häufigsten untersuchten komplementären Medikationen bei OA. Im Handel befinden sich zwei Formen: Glukosaminsulfat und Glukosaminhydrochlorid. Belege zur Wirksamkeit beider Zubereitungen (1'500 mg/Tag) werden im Folgenden separat aufgeführt.

\section{Glukosaminsulfat GS (Wirk-Score 3)}

Ein aktueller Review-Artikel fasst die Ergebnisse von 18 RCTs zusammen, die die Wirksamkeit von GS bei Patienten mit OA untersuchten. Die Anzahl der Probanden in diesen Studien betrug zwischen 30 und 319 Patienten und die Studiendauer bewegte sich zwischen 3 Wochen und 3 Jahren. In 13 RCTs, die GS mit Placebo verglichen, unterschieden sich die Aussagen über Anzahl und Schweregrad unerwünschter Wirkungen bei Patienten unter der Prüfmedikation nicht wesentlich von den Angaben der Patienten unter Placebo. Sieben dieser Studien zeigten, dass GS signifikant wirksamer als Placebo bei der Schmerzlinderung war, und drei von fünf Studien ergaben, dass GS signifikat besser als Placebo im Hinblick auf die Gehfähigkeit oder die Verrichtung täglicher Routinetätigkeiten war.

Zwei weitere Studien (222 Teilnehmer, Behandlungsdauer 2 Jahre; 318 Teilnehmer, Behandlungsdauer 6 Monate) jüngeren Datums prüften die Wirksamkeit von GS in der Therapie von $\mathrm{OA}$ an Hüfte bzw. Knie. Die erste Studie zeigte keine positiven Wirkungen von GS im Vergleich zu Placebo bei der Schmerzlinderung und Funktionsverbesserung, wohingegen die zweite Studie einen signifikanten Vorteil von GS gegenüber Placebo bei der Schmerzlinderung und Funktionsverbesserung zeigte, und sogar eine noch stärkere Wirkung als Paracetamol.

Ein weiterer Review fasste die Ergebnisse von Studien zusammen, die die klinische Wirksamkeit und Sicherheit von GS im Vergleich zu NSAIDs prüfte. Zwei von drei Studien zeigten, dass GS signifikant wirksamer als NSAIDs war bei der Schmerzlinderung, während die dritte Studie zu dem Schluss kam, dass beide Medikationen ähnliche Wirkungen haben. Eine von zwei Studien zeigte, dass GS signifikant besser als NSAID bei der Verbesserung der physikalischen Funktionen war, während die zweite Studie ergab, dass beide Medikationen ähnliche Wirkungen hatten. Drei von vier Studien belegten, dass der Schweregrad unerwünschter Wirkungen bei Patienten unter GS signifikant geringer war als bei Patienten unter NSAIDs.

\section{Glukosaminhydrochlorid GH (Wirk-Score 1)}

In lediglich zwei Studien wurde die Bedeutung von GH in der Therapie von Patienten mit OA am Knie untersucht. In der ersten Studie (118 Teilnehmer, Studiendauer 8 Wochen) berichteten $49 \%$ der randomisiert ausgewählten Patienten, dass sie "sich besser fühlten als zu Studienbeginn". Die gleiche positive Reaktion wurde von $40 \%$ der Patienten unter Placebo berichtet; daraus lässt sich folgern, dass GH bei der Verbesserung OA-assoziierter Symptome dem Placebo nicht signifikant überlegen ist. Es zeigte sich ausserdem, dass GH bei Schmerzlinderung, Morgensteifigkeit und physikalischer Funktion nicht signifikant besser als Placebo abschnitt. In der zweiten Studie (1'583 Probanden, Studiendauer 24 Wochen) wurden Patienten mit OA am Knie randomisiert auf folgende fünf Therapien verteilt: GH (1500 mg/ Tag), Chondroitinsulfat (1200 mg/Tag), beide Medikationen, Celecoxib oder Placebo. Bei den Patienten unter GH oder Chondroitinsulfat verbesserten sich Schmerz, Gelenksteife und physikalische Funktion nicht signifikant im Vergleich zu Placebo. Die einzigen beiden Patientengruppen, bei denen sich eine signifikante Verbesserung der OA-assoziierten Symptome im Vergleich zu Placebo zeigte, waren die Celecoxib-Gruppe und diejenigen Patienten, die GH kombiniert mit Chondroitinsulfat erhalten hatten. In beiden Studien traten unter GH nur leichte Nebenwirkungen auf.

\section{Schlussfolgerung}

Der vorliegende ARC-Report bestätigt eine klare klinische Wirksamkeit von Fischöl bei Rheumatoider Arthritis. Bei Osteoarthritis ergeben sich deutliche Hinweise auf eine klinische Wirksamkeit von Chondroitin und Glukosaminsulfat, die Beweislage zur Wirksamkeit von Glukosaminhydrochlorid ist nicht überzeugend. Die geprüften Medikation werden als sicher eingestuft angesichts nur leichter und seltener unerwünschter Wirkungenen.

Macfarlane G.L. et al.: Complementary and alternative medicines for the treatment of rheumatoid arthritis, osteoarthritis and fibromyalgia. ARC-Report 1/2009. ISBN 978-1-901815-13-9 • www.arc.org.uk/arthinfo/patpubs/6300/6300.asp 